\title{
Photoquenching Indications of a Thermally Triggered Transition Between Two Different EL2 Metastable States in GaAs
}

\author{
P. P. Fávero and J. M. R. Cruz \\ Núcleo de Física Aplicada, Instituto de Física, Universidade de Brasília, Caixa Postal 04455, 70910-900, Brasília, DF, Brazil
}

Received on 4 April, 2005

\begin{abstract}
In this work we report the observation of two different EL2 metastable states in GaAs and the effect of the optical/thermal history of the sample on the behavior of the photoquenching kinetics. In one thermal/optical preparation, the photoquenching curve presented two time constants that have already been interpreted as an indication of two different metastable states. With another preparation, the initial rise in transmittance displayed only one time constant, and we observed that a temperature increase to $83 \mathrm{~K}$ triggered a second photoquenching process. We associated this new photoquenching with a transition from the first to the second metastable state. The experimental data is explained in terms of a new proposal for the microscopic structure of the EL2 complex.
\end{abstract}

Keywords: EL2; GaAs; Photoquenching

\section{INTRODUCTION}

The $E L 2$ is a natural growth defect which has a typical concentration of about $1 \times 10^{16} \mathrm{~cm}^{-3}$ in undoped Liquid Encapsulated Czochralski (LEC) grown gallium arsenide samples. Besides being a donor center, the EL2 presents a metastable state that results from a lattice relaxation induced by a transition with $1.0-1.3 \mathrm{eV}$ photons to an excited state of the center $\left(E L 2^{*}\right)$. After the relaxation, the metastable state becomes optically inactive to photons that have induced the transformation, a phenomenon called photoquenching $[1,2]$. A persistent metastable state can only be achieved when the sample is cooled below 90K.[3] The reverse path, i.e., the total recovery of the $E L 2$ from the metastable state back to the normal state, is achieved when the sample is heated above 120K.[4] This reverse path is also known as thermal recovery.

At first, the EL2 had been associated to a substitutional oxygen due to the proximity of the photoionization energy levels of these two centers.[5]. This model was discarded when the experiments revealed that the EL2 concentration increases when the GaAs is grown on As-rich conditions.[6] Therefore, subsequent proposals included gallium vacancies $\left(\mathrm{V}_{G a}\right)$, arsenic antisites $\left(\mathrm{As}_{G a}\right)$ or arsenic interstitials $\mathrm{As}_{i}$.[5] During the 1980's, experimental and theoretical results indicated that the EL2 could be the isolated $\mathrm{As}_{G a}$, like the model proposed by Chadi and Chang[7], or an association of an arsenic antisite with an arsenic interstitials[8]. Later, evidences were accumulating that the center was more complex and required the presence of an actuator center, responsible for triggering the transition to the metastable configuration of the center.[9] Recently, a three-center complex model has been proposed by Fukuyama et al. [10] that seems to incorporate the necessary features, like the actuator level as well as the metastable state and photoquenching characteristics, but the matter is still open for investigation. In a recent work we presented indications of the existence of two metastable configurations, which seem to be accessed sequentially during the photoquenching process.[11] Furthermore, we showed that, differently from the final metastable state, the intermediate metastable state has a temperature dependent optical absorption cross-section.
In this work we bring an experimental result that shows a thermally triggered transition from the intermediate to the final metastable state $\left(E L 2_{(a)}^{*} \rightarrow E L 2_{(b)}^{*}\right)$. This process seems to imply a strong correlation among the centers in this transition through some kind of cooperative effect. In this respect the mechanism behaves in similar way to the auto-catalytic thermal recovery process recently proposed by Fukuyama and colleagues, except for the fact that it happens in the opposite direction.[10]

\section{EXPERIMENT}

The sample investigated in this work consisted of a nominally undoped $1 \times 1 \times 2 \mathrm{~cm}^{3}$ GaAs block containing $1.45 \times$ $10^{16} \mathrm{~cm}^{-3} E L 2$ centers, as determined by optical absorption spectroscopy[12], and a carbon concentration of the order of $1 \times 10^{16} \mathrm{~cm}^{-3}$, estimated from FFT-IR absorption.[13] The spectral analysis revealed that essentially all EL2 were in the normal state at room temperature. The lack of an appreciable concentration of $E L 2^{+}$, together with a resistivity of $6.2 \times 10^{6}$ $\Omega \mathrm{cm}$, may be an indication of a residual n-type doping.

The experiment consisted of two cooling-photoquenchingheating cycles, differing from each other by the sample thermal preparations, as detailed ahead. The transition of the $E L 2$ to its metastable state was produced by an InGaAs laser beam at $\lambda=1.05 \mu \mathrm{m}(1.18 \mathrm{eV})$. The beam was focused at the sample surface and the intensity was adjusted by the insertion of calibrated filters. The temperature was controlled by placing the sample in a cold finger liquid-nitrogen-flow optical cryostat, and was monitored by two sensors mounted on the top and bottom sides of the sample. Since the beam was positioned at the center of sample, the temperature at the irradiated spot was estimated to be the average of these two sensor readings.

In the first optical/thermal sample preparation, we have repeated some cycles of cooling the sample, producing the photoquenching effect and heating it up to $150 \mathrm{~K}$. After this preparation, the sample was cooled down to $77 \mathrm{~K}$ in the dark and it was then subjected to the cw laser. In Fig. 1, we present the photoquenching curve obtained. The transmittance rise dis- 


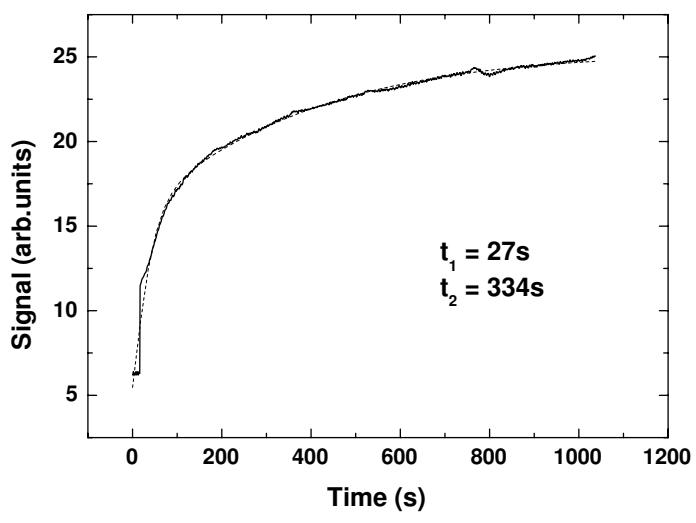

FIG. 1: Photoquenching curve obtained after the first sample preparation (see text). The transmittance increases due to transition to the metastable state and displays two time constants.

plays two time constants (compare with the first rise in Fig. 2). After the complete photoquenching process the temperature was increased up to $300 \mathrm{~K}$.

In the second optical/thermal sample preparation procedure, the sample was maintained at $300 \mathrm{~K}$ for 5 minutes after the first experiment was completed, and cooled down again in the dark to $77 \mathrm{~K}$ with a very high cooling rate $\left(1-2 \mathrm{~K} \mathrm{~s}^{-1}\right)$. Next, it was subjected to the laser beam. The new photoquenching curve obtained can be seen in Fig. 2. The initial transmittance rise, differently from the behavior displayed in Fig. 1, seems to have only one time constant. After over 1000s of strong illumination, the temperature was increased up to $83 \mathrm{~K}$ and brought down to $71 \mathrm{~K}$, as shown in the lower curve of Fig. 2. The curve shows a sudden increase in transmittance which was apparently triggered by the heating of the sample under illumination. Despite the immediate reduction in temperature, after the second photoquenching started, the increase in transmittance continued up to a higher stable value.

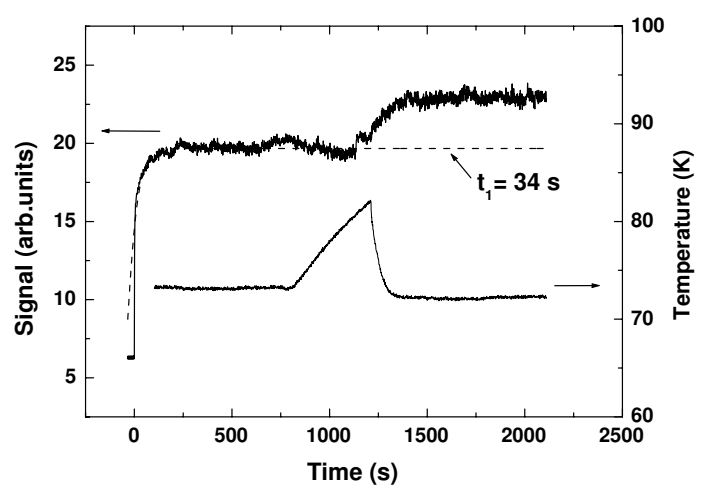

FIG. 2. Photoquenching curve obtained after the second sample preparation (see text). Differently from Fig. 1, the initial rise in transmittance displays only one time constant. The lower curve shows the applied temperature changes. A momentary rise in temperature up to $83 \mathrm{~K}$ triggered a transition to another metastable phase.

\section{ANALYSIS AND DISCUSSION}

In the absence of $\mathrm{EL2}^{+}$and other absorbing defects, the transmittance is essentially related to the population of $E L 2$ in the normal state $\left(E L 2^{0}\right)$, and can be expressed by

$$
T=\frac{(1-R)^{2} e^{-\alpha L}}{1-R^{2} e^{-2 \alpha L}}
$$

where $R$ is the sample surface reflectivity and $\alpha$ is the absorption coefficient. If there is more than one absorbing defect, the absorption coefficient is a sum of their individual absorption coefficients. Therefore it depends on the product of their concentration and their absorption cross-sections. The shapes of the photoquenching curves in Figs 1 and 2, reveal two time constants and one time constant, respectively. For this reason, we have fitted the transmittance curves with the following expression:

$$
T=C \exp \left(-a_{1} e^{-\frac{t}{t_{1}}}-a_{2} e^{-\frac{t}{t_{2}}}\right)
$$

where $C, a_{1}, a_{2}, t_{1}$, and $t_{2}$, are the fitting constants for the case of Fig. 1, and $a_{2}$ and $t_{2}$ were neglected in the case of Fig. 2.

In a recent work we suggested that the two time constants, which are present at the first photoquenching curve, are associated with two sequential EL2 transitions, from the normal to an intermediate metastable state $\left(E L 2^{0} \rightarrow E L 2_{(a)}^{*}\right)$, and from this to a final metastable state $\left(E L 2_{(a)}^{*} \rightarrow E L 2_{(b)}^{*}\right)$. This hypothesis was based on the different temperature dependence of the absorption cross-section presented by the intermediate state that differs from the behavior of the final metastable state.

The basic $E L 2^{0}$ state in our proposal is shown in Fig. 3. The $\mathrm{As}_{G a}$, initially in the position labeled as (1), is responsible for the metastability, whereas the $\mathrm{Ga}_{A s}$ controls the transition to the metastable state via the coulomb interaction with the neighboring $\mathrm{As}_{G a}[10]$. Instead of a $\mathrm{V}_{A s}$, as proposed by Fukuyama et al[10], our structure incorporates a $\mathrm{V}_{G a}$, which is more easily justified considering that it leads to an Asrich structure. In the following, we have labeled this state as $E L 2_{(1)}^{0}$ to differentiate it from the similar state $E L 2_{(2)}^{0}$ depicted in Fig.3e, where the $\mathrm{As}_{G a}$ is in the position labeled as (2). As already proposed[10], the transition to the metastable state begins with a photoexcitation of the $\mathrm{As}_{G a}$ with a charge transfer from the $\mathrm{Ga}_{A s}$, resulting in the breaking of the $\mathrm{As}_{G a^{-}}$ $\mathrm{Ga}_{A s}$ bond (Fig. 4b). This effect loosens the $\mathrm{As}_{G a}$ atom and the excited states relaxes via an Jahn-Teller effect to the configuration shown in Fig. 3c.[7, 14]. Next, we propose that, under a new photoexcitation, a further migration of the As atom is possible. It moves to a position along another $<111>$ direction, towards the $\mathrm{V}_{G a}$. The bonds are then reestablished with the nearest neighbor atoms of this site and the released ones constitute a new $\mathrm{V}_{G a}$ site, located where the $\mathrm{As}_{G a}$ was originally. As the temperature rises, the As atom in this latter configuration has two choices: either it makes the reverse path back to (a) $\left(E L 2_{(1)}^{0}\right)$ or it relaxes to the position described by 
(e) $\left(E L 2_{(2)}^{0}\right)$ in Fig. 3. The energy barriers for the two paths should be slightly different since their electronic environments are not equal.

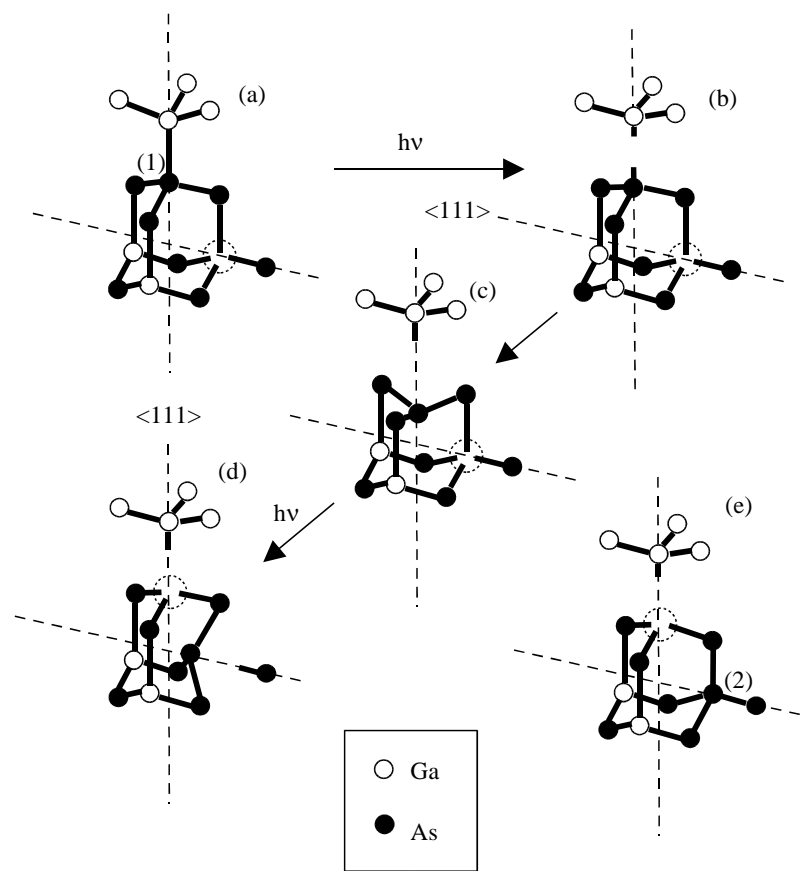

FIG. 3. Different stages of the $E L 2$ center. (a) The normal $E L 2^{0}$; (b) After photoexcitation there is a charge transfer between the $G a_{A s}^{-}$and the $A s_{G a}^{+}$that leads to the $G a_{A s}-A s_{G a}$; (c) The structure relaxes with the motion of the As atom and the flipping down of the three remaining bonds; (d) After a new photoexcitation, the atom migrates to an equivalent site along a different $\langle 111\rangle$ direction towards the $V_{G a}$, and rearranges the bonds with the nearest neighbor atoms of this site. This corresponds to the motion of the vacancy to the original $A s_{G a}$; (e) This configuration may be one of the possibilities of a thermal recovery.(see text).

The two possibilities for the $\mathrm{As}_{G a}$ in this structure can lead to two final states during the recovery in a non-adiabatic heating process. This may explain why the properties of system are so dependent on its thermal history. The initial conditions can be reestablished by heating the system up to $300 \mathrm{~K}$ for a prolonged time. Therefore we expect the final configuration (e) $\left(E L 2_{(2)}^{0}\right)$ to have a higher energy than the configuration (a) $\left(E L 2_{(1)}^{0}\right)$ to where the system relaxes at high temperatures. These conclusions are summarized in the proposed configurational coordinate diagram in Fig. 4.

The fitting of the data in Fig. 1 reveals two time constants $\left(t_{1}=27 \mathrm{~s}\right.$ and $\left.t_{2}=334 \mathrm{~s}\right)$, whereas the first rise shown in Fig. 2 has only one $\left(t_{1}=34 \mathrm{~s}\right)$. Based on our proposal for the microscopic structure, the first sample preparation seems to have lead the sample to the $E L 2_{(1)}^{0}$ state (see Fig. 3a). The first time constant $\left(t_{1}\right)$ is related to the transition to the intermediate metastable state $E L 2_{(1)}^{0} \rightarrow E L 2_{(a)}^{*}$ and the longer time constant $\left(t_{2}\right)$ is related to the second transition to the final metastable state $\left(E L 2_{(a)}^{*} \rightarrow E L 2_{(b)}^{*}\right)$.
The data in Fig. 2 can be explained in terms of our microscopic model by considering that, during the second preparation, a charge gets trapped in the gallium vacancy $\left(\mathrm{V}_{G a}\right)$ (see Fig. 3a). The assumption is that this charge impedes the motion of the As atom in the transition from the metastable configuration (a) to (b). Therefore, the first time constant corresponds to the transition $E L 2_{(1)}^{0} \rightarrow E L 2_{(a)}^{*}$. The system would remain locked in the meatastable configuration (a) while the $\mathrm{V}_{G a}$ site is charged. The second rise in the transmission would only happen after the charge from the $\mathrm{V}_{G a}$ site is released by the heating to $83 \mathrm{~K}$, allowing the transition $E L 2_{(a)}^{*} \rightarrow E L 2_{(b)}^{*}$ to occur.

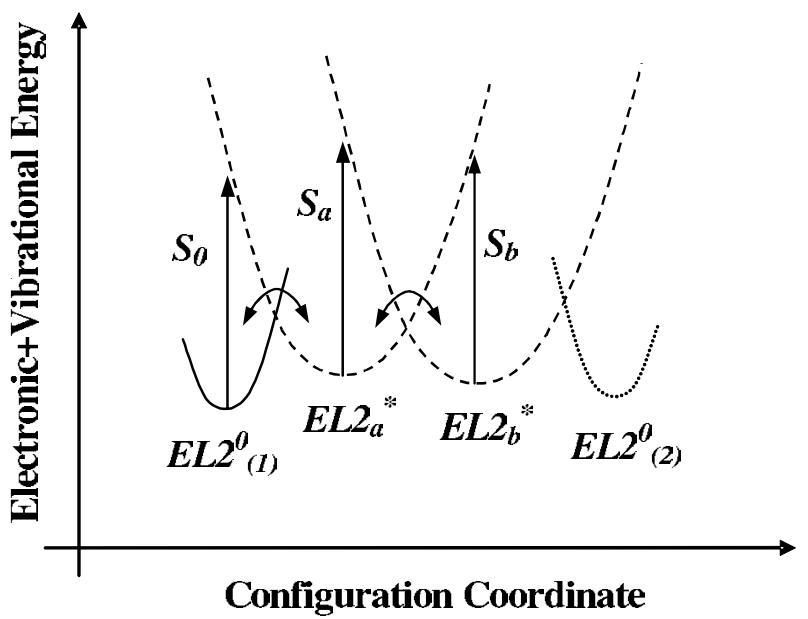

FIG. 4. Configurational coordinate diagram proposed for the EL2 system, incorporating an intermediate state. The system can pass from the intermediate $E L 2_{a}^{*}$ to the final $E L 2_{b}^{*}$ state by optical absorption. There are two different configurations for the normal state, labeled $E L 2_{(1)}^{0}$ and $E L 2_{(2)}^{0}$. The first one is considered to be the lowest energy state. The curves corresponding to the conduction and valence bands were omitted for clarity.(see text).

During the heating process to $300 \mathrm{~K}$, a fraction of the $E L 2$ might have ended in the configuration $E L 2_{(2)}^{0}$ and, without giving enough time for the system to find the equilibrium configuration, the non-adiabatic fast cooling process may have locked some of the EL2 centers in this alternative state. Therefore, the first transmittance rise in Fig. 2 may contain a contribution from the optical photoquenching transition from this state directly to the final $E L 2_{(b)}^{*}$ state. The superposition of the two fast transitions, i.e., from $E L 2_{(1)}^{0} \rightarrow E L 2_{(a)}^{*}$ and $E L 2_{(2)}^{0} \rightarrow E L 2_{(b)}^{*}$ may explain the small differences observed in the time constant $t_{1}$ in Figs. 1 and 2.

\section{CONCLUSION}

The results of this work emphasise the importance of a very controlled sample preparation when the objective is the study the EL2 defect. The sample thermal history is fundamental to the assessment of both the metastable and normal states. Besides confirming the two-time constants already reported 
in a separate work with a different set of data and temperature [11], this work indicates the possibility of the locking of the intermediate metastable state with, what we believe to be, a possible charge state. The triggering of this intermeditate transition with a small temperature increase, and the fact that it keeps proceeding despite a subsequent temperature reduction, seems to indicate a cooperative interaction mechanism between centers, similarly to what have been recently suggested in the case of the thermal recovery.[10]. It can also be infered from the measured time constant that the cross-section for the transition from the fundamental to the metastable state is about ten times larger than that for transition from the intermediate to the final metastable state.
[1] G. M. Martin, Appl. Phys. Lett. 39, 747 (1981)

[2] D. Bois and G. Vincent, J. Phys. Lett. 38, 351 (1977).

[3] G. Vincent, D. Bois, and A. Chantre, J. Appl. Phys. 53, 3643 (1982).

[4] I. K. Kristensen and S. Moller, Solid State Comm. 99, 751 (1996).

[5] G. A. Baraff, in Deep Centers in Semiconductors ed. S. Pantelides (Gordon and Breach Sci.Pub., New York, 1986)

[6] D. E. Holmes, R. T. Chen, K. R. Elliott, and C. G. Krkpatrick, Appl. Phys. Lett. 40, 46, (1982).

[7] D. J. Chadi and C. Chang, Phys. Rev. Lett. 60, 2187 (1988).

[8] B. K. Meyer, D. M. Hofmann, J. R. Niklas, and J.-M. Spaeth,
Phys. Rev. B 36, 1332 (1987)

[9] J. Jimenez, A. Alvarez, and M. Chafai, Phys. Rev. B 50, 14112, (1994).

[10] A. Fukuyama, T. Ikari, Y. Akashi, and M. Suemitsu, Phys. Rev. B. 67, 113202 (2003).

[11] P. P. Fávero and J. M. R. Cruz, Eur. Phys. J.B. 47, 363 (2005).

[12] P. Silveberg, P. Omling, and L. Samuelson, Appl. Phys. Lett. 52, 1689 (1988).

[13] M. R. Brozel, E. J. Foulkes, R. W. Series, and D. T. J. Hurle, Appl. Phys. Lett. 49, 11 (1986).

[14] J. Dabrowski and M. Scheffler, Phys. Rev. B 40, 10391 (1989). 